\title{
SEMBLANZA
}

\section{Carlos Battilana: Profesor, Gestor, Amigo}

\author{
Carlos Battilana: Professor, Manager, Friend
}

\author{
José Pacheco, Eleazar Aliaga \\ Facultad de Medicina, Universidad Nacional Mayor de San Marcos. Lima, Perú.
}

\begin{abstract}
Resumen
El Comité Editorial de Anales ha perdido a uno de sus miembros más connotados. Brillante docente de nuestra Facultad, Carlos Alberto Battilana Guanilo (19452009) supo transmitir los conocimientos y atraer la atención de sus auditorios, de jóvenes estudiantes o de contemporáneos ya no tan jóvenes. Interesó a sus alumnos en la senda de la capacitación permanente y en la investigación. Por otro lado, comprometió a médicos distinguidos a conformar y liderar grupos con interés en la ciencia-amistad. Su vocación docente lo vinculó a facultades de medicina y academias y sociedades científicas, en donde coordinó cursos y congresos de grato recuerdo. Su producción científica la dedicó a la nefrología, inmunología, cáncer, costos en el tratamiento médico. Su capacidad gestora y de liderazgo presente desde su época de estudiante, le permitió llegar a ser director regional de un laboratorio farmacéutico de mucho prestigio, organizar una facultad de medicina y luego tener el cargo de decano de la facultad de ciencias de la salud de dicha universidad privada. Carlos fue elemento importante para que Anales alcanzara un sitial de privilegio entre las revistas biomédicas peruanas. En la semblanza que publicamos tratamos de resumir apretadamente la trayectoria de Carlos Battilana, semanas después de su partida sin retorno.

Palabras clave: Battilana, Carlos; estudiantes de medicina; docente médico; nefrología; medicina interna.
\end{abstract}

\begin{abstract}
Anales' Editorial Committee has lost one of its most renowned staff members. Brilliant professor of our Faculty of Medicine, Carlos Alberto Battilana Guanilo (1945-2009) knew how to transmit knowledge and appeal audiences' attention, either in young students or not so young contemporary fellows. He got his students interested in the paths of permanent learning and investigation. On the other hand, he committed distinguished physicians and professors to constitute and lead science-friendship interested groups. His vocation in teaching bound him to faculties of medicine and scientific academies and societies, where he coordinated courses and meetings that are pleasantly recalled. His scientific production was devoted to nephrology, immunology, cancer, medical treatment costs. His capacity of management and leadership present since being a student, allowed him to become regional director of a prestigious pharmaceutical laboratory, to organize a faculty of medicine and then to be the dean of the faculty of health sciences of such private university. Carlos was an important element for Anales de la Facultad de Medicina to reach a privileged place among Peruvian biomedical journals. In this biographical sketch we try to summarize Carlos Battilana's brilliant career, weeks after his departure with no return.
\end{abstract}

Key words: Battilana, Carlos; students, medical; faculty, medical; nephrology; internal medicine.
La Facultad de Medicina de San Fernando y Anales de la Facultad de Medicina han perdido a uno de sus miembros y profesores más valiosos. Carlos Alberto Battillana Guanilo nació en Lima, un 14 de agosto del año 1945. Sus padres fueron Carlos Battilana, de origen genovés, y Blanca Guanilo, nacida en Pacasmayo. Realizó sus estudios escolares en el Colegio Claretiano, donde fue el mejor alumno de su promoción, preludiando su futuro promisorio. Su innata vocación de servicio le llevó a elegir la profesión médica, eligiendo estudiar en la Facultad de Medicina de San Fernando, de la Universidad Nacional Mayor de San Marcos, en donde ingresó el año 1963 (usando en su primer acercamiento un sombrero tejano), y terminó la carrera el año 1971, en una promoción donde encontramos a varios líderes actuales de la medicina y de distinguidos docentes de nuestra Universidad.
Carlos Battilana fue desde muy joven un guía (figura 1), que impulsaba y desarrollaba nutrida actividad científica, social e institucional. Prueba de ello es su participación como dirigente estudiantil, cuando estimulaba a las masas de estudiantes con sus vibrantes y amenos discursos, y posteriormente como miembro del Consejo de Facultad, en San Fernando, durante los años 1988, 1989. Al ingresar a su actividad hospitalaria, como estudiante conoce al Dr. Pedro Domínguez Mejía (figura 2), quien fue su profesor y guía, sintiendo una gran admiración por él. Prueba de ello es su inclinacion inicial por la cardiología, lo cual se demuestra con la Tesis que realizó, en la cual Domínguez se constituyó en su tutor. A su retorno de los EE UU, esta admiración se convirtió en amistad, al reconocer el maestro la calidad que como médico y profesor mostraba Carlos.

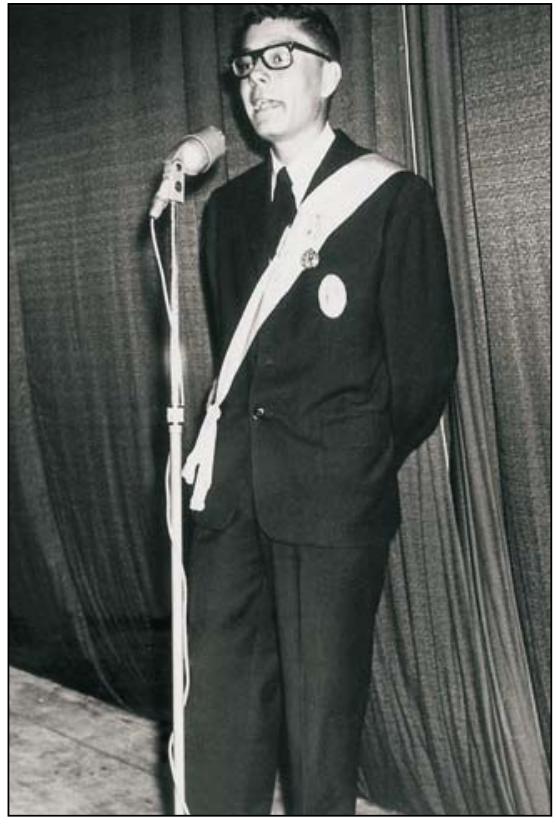

Figura 1. Carlos Battilana en una fotografía tomada en su juventud. 


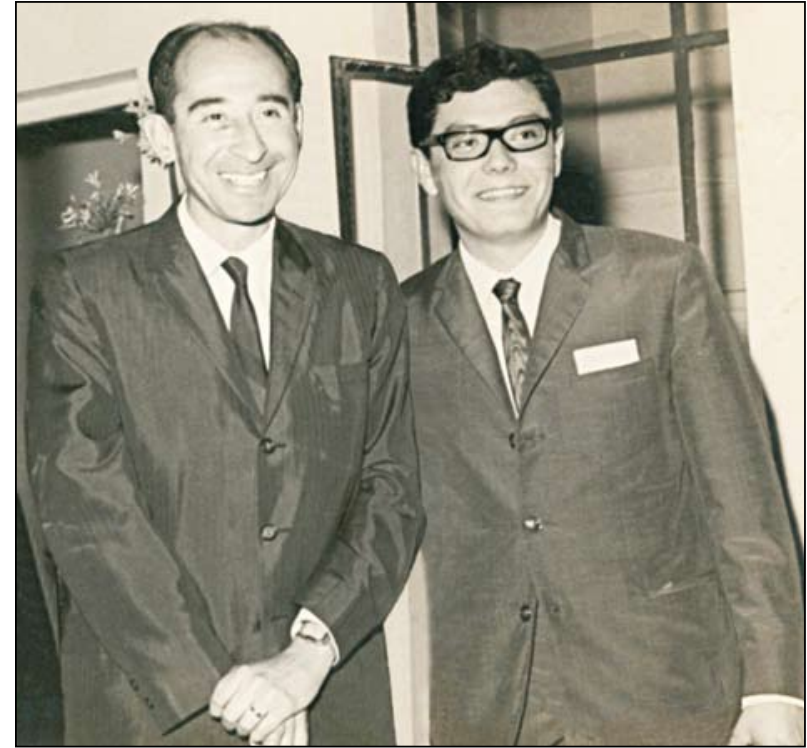

Figura 2. Carlos Battilana con el Dr. Pedro Domínguez, quien lo relacionó con la cardiología.

Se graduó de médico el año 1971, presentando la tesis Estudio clínico de enfermedad miocárdica primaria (cardiomiopatía). Observaciones en 26 casos. La investigación, por su calidad científica, obtuvo el Premio a la Mejor Tesis, otorgado por el laboratorio Merck Sharp \& Dohme. Ese año partió a los Estados Unidos de América del Norte, para realizar sus estudios de posgrado, ingresando al Hospital Union Memorial, afiliado a la Universidad de Johns Hopkins, y luego al Hospital Henry Ford, institución formadora de muchos médicos peruanos sobresalientes, donde llegó a ser Jefe de Residentes de Medicina Interna. Aunque su primera orientación había sido la cardiología, pronto encontró la que sería la especialidad de sus amores. En 1974, fue admitido en la Universidad de Stanford, para especializarse en nefrología, dedicándose posteriormente a la enseñanza e investigación de la fisiología y patología renal.

A su regreso al Perú, en 1978, se volcó a la docencia, ingresando como Profesor Auxiliar a la Facultad de Medicina de San Fernando y realizando la enseñanza universitaria en las diferentes salas del hospital Dos de Mayo. Se le encontraba por los pasillos de este hermoso hospital buscando conjuntamente con sus alumnos los pacientes que pudiesen ilustrar de la mejor manera los diferentes síndromes de la especialidad. Posteriormente, se incorporó a la recién creada Clínica San Borja, como médico internista y nefrólogo. Desde entonces se le ve multiplicándose en sus labores del hospital y de la clínica, escuchándose en la Sala Santo Toribio del Hospital Dos de Mayo el sonido peculiar de los pocos beepers de entonces y saliendo apuradamente para buscar un teléfono público y responder al llamado. Poco tiempo después, con la Dra. Vilma Santivañez crea el Centro de Hemodiálisis de la Clínica San Borja, que se constituyó en un centro de referencia y excelencia de la nefrología en el Perú.

Carlos vivía con pasión la docencia y la investigación. Como docente, su inicio data de ser Jefe de Prácticas de neuroanatomía. A su retorno al Perú, le ocurrió lo que a muchos de quienes retornábamos al terruño. No había plazas hospitalarias disponibles y los concursos se aplazaban indefinidamente. Para suerte de la medicina peruana, Carlos se dedicó a la enseñanza, desempeñándose en los cursos de Semiología Clínica -hoy llamado Introducción a la Clínica- y del Capítulo de Nefrología del Curso de Medicina II, hoy Medicina Interna. Con el trabajo Estudio metabólico en 100 pacientes con urolitiasis con especial énfasis en la diferenciación de las hipercalciurias, obtuvo el grado de Doctor. En el año 1991, Battilana ascendió a la categoría de Profesor Principal de San Fernando.

Al mismo tiempo, y a lo largo de los años, realizó intensa investigación científica, publicando artículos en diferentes revistas e innumerables presentaciones en congresos nacionales e internacionales. Fue promotor de la investigación entre los estudiantes de medicina. De esta manera, a partir de 1990 y durante 8 años consecutivos fue designado Asesor del Congreso Nacional de Estudiantes de Medicina, que se realiza anualmente en Lima o en provincias. No satisfecho con lo logrado hasta el momento, ese año 1991 funda IPICA, el Instituto Peruano de Investigación Clínica Aplicada, a través del cual promovió y brindó apoyo económico a jóvenes investigadores; la institución, además, ofrecía cursos, seminarios y talleres de educación médica continua.

Participó en un sinnúmero de cursos de posgrado, nacionales e internacionales (figura 3), así como en cursos auspiciados por el Colegio Médico; se desempeñó como coordinador de varios de ellos, uno de los cuales nos dejó huella. En el Curso "Manejo de los pacientes con alteraciones hidroelectrolíticas y del equilibrio ácido base", que dio origen al libro del mismo tema, llamó la atención la metodología utilizada, innovadora para ese entonces, que consistía en dictar casos clínicos a auditorios que tenían un promedio de 200 participantes e invitar a los asistentes a desarrollar los problemas, solicitando luego las soluciones.

En 1995 pasó a ser Director Médico de Productos Roche Q.F.S.A. y, dada su alta productividad gerencial, llegó a tener el cargo de Director Regional, que desempeñó hasta su fallecimiento. En el año 2005 creó el Área de Investigación Clínica de Roche, en la que gracias a su destacada labor, en agosto de 2009, obtuvo el primer puesto regional en investigación clínica.

Fue miembro de numerosas sociedades científicas e instituciones profesionales, entre ellas de la Academia Nacional de Medicina -de la que fue Académico de Número-, del American College of Physicians y de la Sociedad Peruana de Medicina Interna, de la que llegó a ser Presidente y Presidente del Comité 


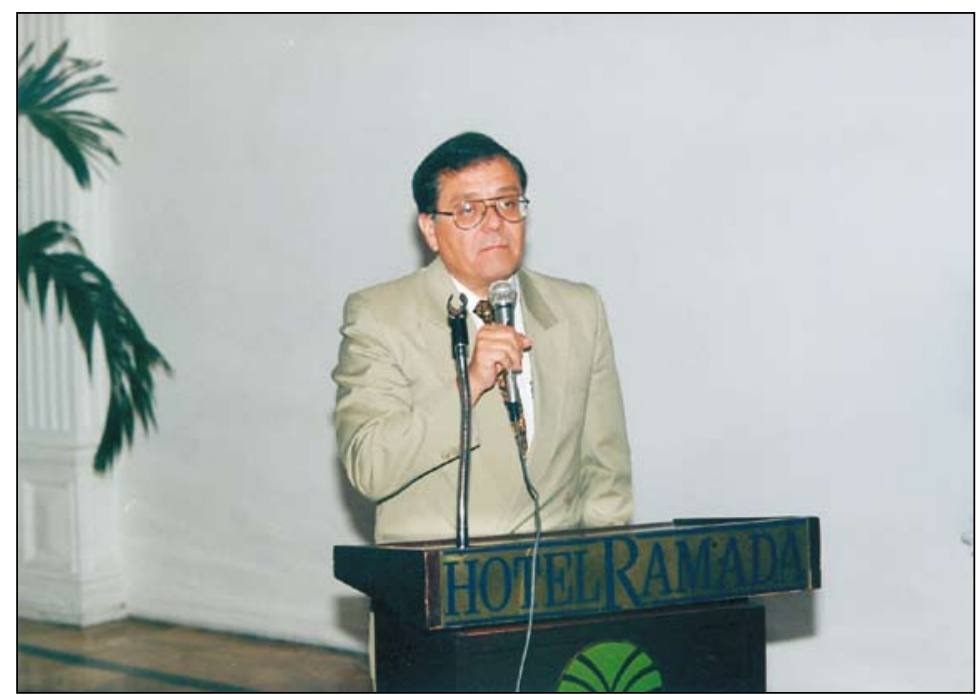

Figura 3. Carlos Battilana, concentrado, en una de sus ilustradas y amenas charlas.

Organizador del Congreso Internacional de Medicina Interna, en el año 1998. Con relación al fellowship obtenido por pocos médicos peruanos en el American College of Physicians-American Society of Internal Medicine, se recuerda el compromiso de ellos para con la institución, en "donde los nuevos convocados deberían ........ asumir la decisión de luchar contra las grandes amenazas del ejercicio de la medicina en el mundo actual", principalmente los problemas en la calidad de atención médica y la seguridad de los pacientes, entre otros ${ }^{(1)}$, compromisos que Battilana tomó como suyos y los desarrolló en el país.

Una anécdota que nos contó Carlos en la inauguración de un Curso de la Sociedad Peruana de Medicina Interna, fue cuando él -Miembro del Comité Ejecutivo de la International Society of Internal Medicine (ISIM) - y Raúl Salazar -Presidente de la Sociedad Peruana de Medicina Interna- viajaron a Vilnius, capital de Lituania, a la reunión de la junta Directiva de ISIM. En esa oportunidad, Israel renunció a la organización del Congreso Internacional de Medicina Interna - ISIM 98. Con toda seguridad y conociendo la habilidad que tenía Carlos en sus fundamentaciones, y, luego de conocer otras propuestas, se reconoció al Perú como una óptima alternativa para suceder a Jerusalén. Este XXIV Congreso Internacional de Medicina
Interna finalmente se realizó en Lima, en noviembre de $1998^{(2)}$. Más adelante, en el año 2006, la Sociedad de Medicina Interna de Polonia le otorgó la Medalla de Honor al Mérito, por su desempeño y eficiencia en medicina.

Carlos nos acompañó en Anales de la Facultad de Medicina desde el volumen 61 número 4, octubre-diciembre 2000, cuando el Dr. Fausto Garmendia (3), entonces Decano de la Facultad de Medicina, invitó al Comité Editorial actual para mantener el lugar importante que Anales había tenido a través de sus más de 90 años de existencia y que la gestión del Dr. José Piscoya había retomado desde 1995. Desde siempre, Carlos nos sugería rutas a seguir para perfeccionar la editorial y el temario. Opinó sobre formatos, carátulas, contenido, índices y otros y se sintió sumamente feliz cuando el Vicerrectorado de Investigación de la Universidad Nacional Mayor de San Marcos nos otorgó el premio al Mérito Científico 2006. Por supuesto, fue artífice en el planeamiento de los festejos de los 90 años de Anales, celebración que contó como actividades culminantes el acto académico en la Casona de San Marcos, el 17 de noviembre de 2008, y un Número Especial $90^{\circ}$ Aniversario de Anales, volumen 69, suplemento 2, noviembre $2008^{(4)}$.

En las reuniones del Comité Editorial, Carlos nos traía la alegría y la chanza sana e iniciaba la discusión de las novedades del momento en el país. Y luego de un brindis y los chistes y bromas hasta que llegaran todos, se iniciaba la sesión del Comité, en la que él gustaba conocer nuestros pensamientos y luego opinaba y sugería, tratando siempre de tomar lo bueno de las partes para expresar su opinión, dando soluciones a los problemas y motivando la satisfacción y asentimiento de los miembros del Comité.

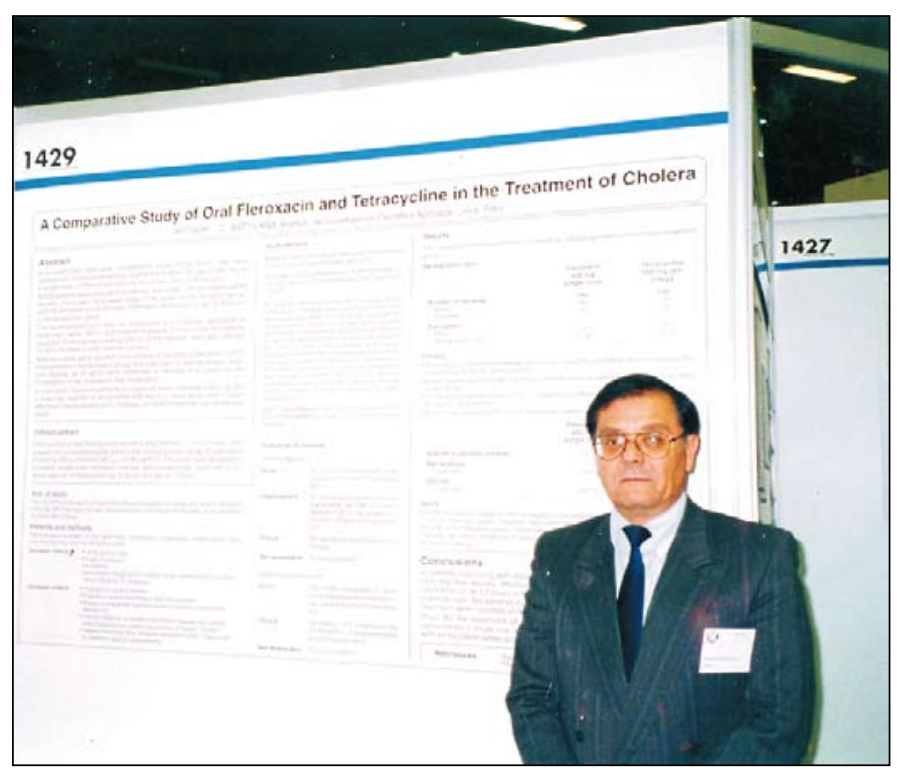

Figura 4. Battilana en la presentación de un póster sobre tratamiento del cólera. 


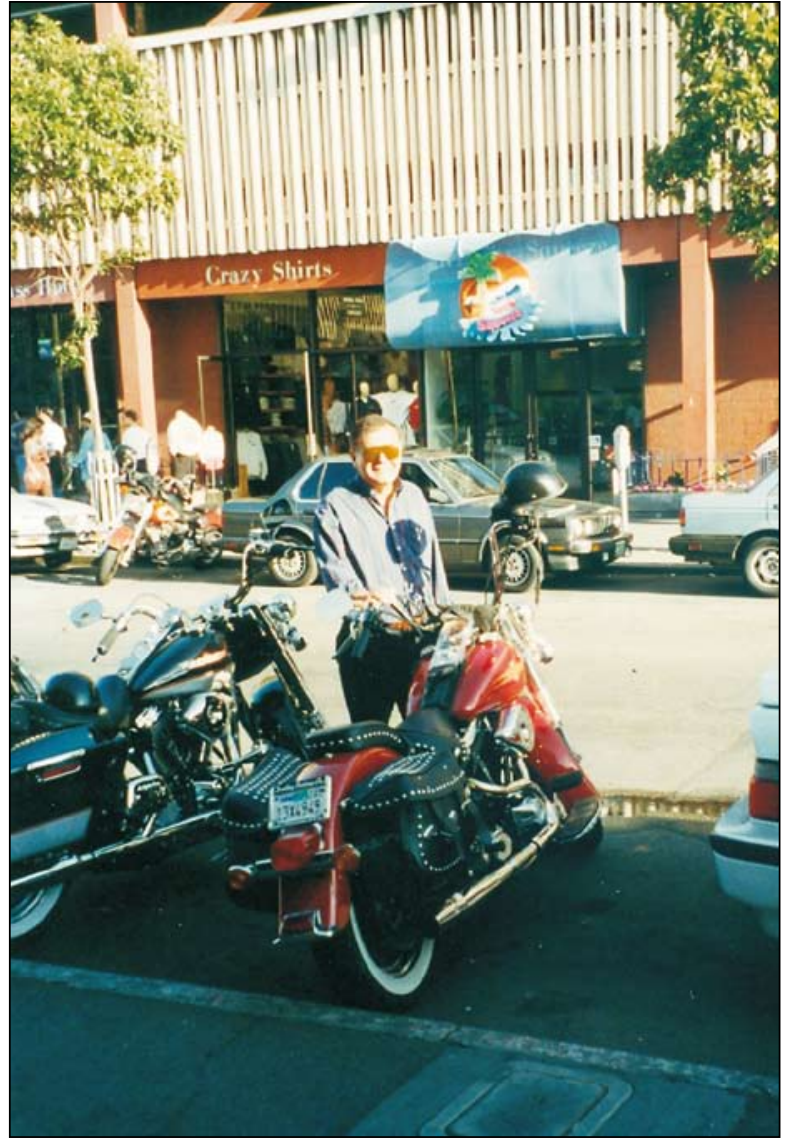

Figura 5. Una de las pasiones de Carlos Battilana eran las motocicletas.

Tuvimos oportunidad de escucharlo en muchas charlas en la Facultad y en reuniones institucionales, muchas promovidas por el Laboratorio en el cual era Director Médico y más adelante Director Regional. Tenía un amplio conocimiento

de la medicina, captaba la atención del público -en especial del más joven-, y dueño de un humor exquisito y de un dominio de escena que envidiarían los actores más connotados, desarrollaba sus charlas, mostrando hermosas gráfi-

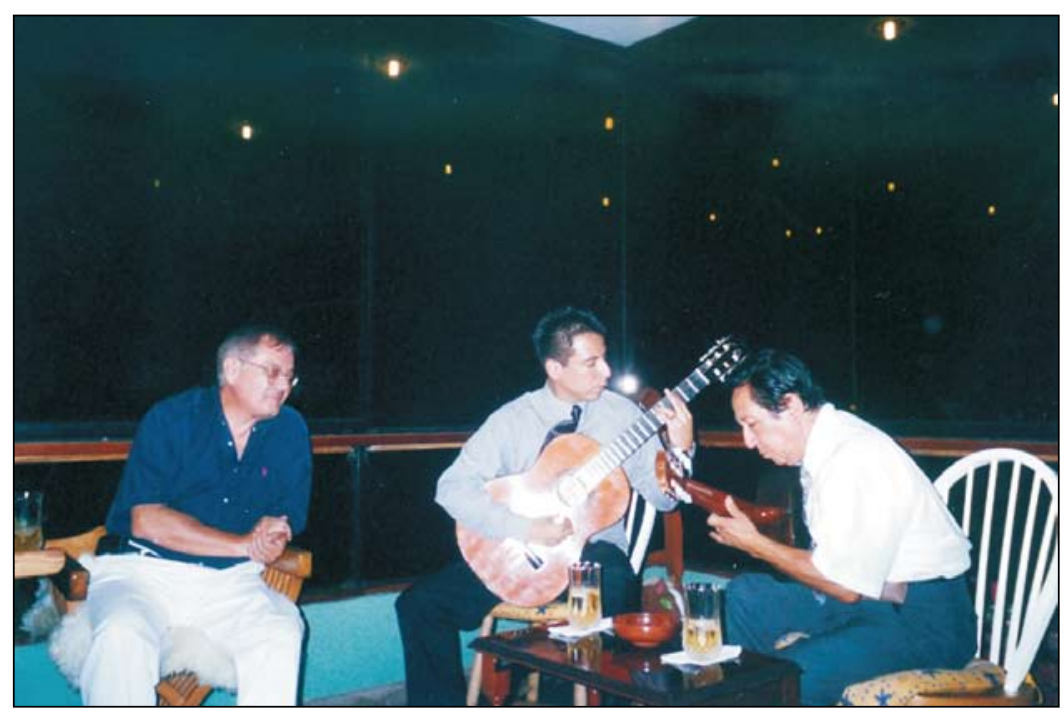

Figura 6. Carlos Battilana en una de las recordadas tertulias de música y amistad. cas y sabía introducir oportunamente ocurrencias y algazaras que amenizaban estas reuniones docentes. Hemos viajado con él dentro y fuera del país, siempre preocupado en el perfeccionamiento de los líderes de especialidades médicas en temas poco vertidos en nuestro medio. Sabía priorizar los auspicios y siempre estuvo dispuesto a colaborar con San Fernando. También hemos tenido la suerte de compartir actividades institucionales con él, como en la Sociedad Peruana de Hipertensión Arterial, o en las actividades que él propulsó en la Sociedad Peruana de Medicina Interna, donde organizó muchos cursos orientados a la gente joven.

Tuvo una importante producción científica. Su primera publicación en Anales fue en el año 1971, de su tesis Estudio clínico de enfermedad miocárdica primaria (cardiomiopatía). Observaciones en 26 casos ${ }^{(5)}$. En PubMed hemos hallado 10 trabajos, publicados en revistas como Journal of Clinical Investigation y American Journal of Physiology, en los que Battilana presenta, entre los años 1977 y 1991, trabajos de investigación sobre riñón y asa de Henle, con nefrólogos norteamericanos ${ }^{(6-9)} \mathrm{y}$, desde 1988, trabajos relacionados a insuficiencia y transplante renal ${ }^{(10-12)}$, así como, tres publicaciones sobre cólera y su manejo ${ }^{(13-15)}$ (figura 4). Muchos de sus trabajos los encontramos en la revista Diagnóstico, de la Fundación Instituto Hipólito Unanue. Observamos en las referencias de dicha revista investigaciones de Battilana sobre nefropatía diabética ${ }^{\left({ }^{16)}\right.}$, presión arterial y manejo medicamentoso ${ }^{(17,18)}$, linfocito B y artritis reumatoide ${ }^{(19)}$, terapia adyuvante del cáncer de mama ${ }^{(20)}$ y varios artículos sobre análisis fármaco-económico (21-27) en tratamientos para el cáncer, la artritis reumatoide, insuficiencia renal, hepatitis $\mathrm{C}$, tema poco tratado por nuestros investigadores y de gran impacto en la evaluación de costos en medicina.

Un amante de su vida familiar, no la descuidó, y tampoco a sus amigos, a pesar del intenso trajín dentro y fuera del país, incluyendo la organización de la Escuela de Medicina y posteriormente el Decanato de la Facultad de Ciencias de la Salud de la Universidad Peruana de Ciencias Aplicadas, y su membresía 
en la Asociación Peruana de Facultades de Medicina, en estos casos, siempre en su afán por promover el mejoramiento de la calidad de la educación médica. Con su esposa Sandra invitaba a sus amigos a tertulias amenas y festivas, preparaba sus propias parrillas, nos contaba de motocicletas (figura 5) y cantaba y bailaba (figura 6), y siempre partíamos con una sonrisa y con algo nuevo aprehendido. Sus hijos Carlos Fernando, Andrea y Antonielli muestran los genes paternos en el perfeccionamiento de sus destinos profesionales.

Carlos Battilana dejó de existir el 15 de setiembre de 2009. Lo recordaremos siempre y el Comité Editorial de Anales acordó que su silla no fuera ocupada, en homenaje al profesor, investigador y amigo.

\section{REFERENCIAS BIBLIOGRÁFICAS}

1. Pamo-Reyna OG, Editorial. ACP-ASIM Fellowship: La confraternidad del anillo. Rev Per Soc Med Intern. 2002;15(3):122-3.

2. Crónicas de la Sociedad. Bol Soc Per Med Interna [Internet]. 1997 [citado el 10 de diciembre de 2009];10(3). Disponible en: http://sisbib.unmsm. edu.pe/bVrevistas/spmi/v10n3/cron_socied.htm

3. Garmendia F. Editorial. Anal Fac med. 2000;64(4):263-4.

4. Blanco E. Editorial. Anal Fac med. 2008;69(Supl 2):5.

5. Battilana C. Estudio clínico de enfermedad miocárdica primaria (cardiomiopatía). Observaciones en 26 casos. Anal Fac med. 1971;54:262.

6. Johnston PA, Battilana CA, Lacy FB, Jamison RL. Evidence for a concentration gradient favoring outward movement of sodium from the thin loop of Henle. J Clin Invest. 1977;59(2):234-40.

7. Gelbart DR, Battilana CA, Bhattacharya J, Lacy FB, Jamison RL. Transepithelial gradient and fractional delivery of chloride in thin loop of Henle. Am J Physiol. 1978;235(3):F192-8.
8. Battilana CA, Dobyan DC, Lacy FB, Bhattacharya J, Johnston PA, Jamison RL. Effect of chronic potassium loading on potassium secretion by the pars recta or descending limb of the juxtamedullary nephron in the rat. J Clin Invest. 1978;62(5):1093-103.

9. Battilana C, Zhang HP, Olshen RA, Wexler L, Myers BD. PAH extraction and estimation of plasma flow in diseased human kidneys. Am J Physiol. 1991;261 (4 Pt 2):F726-33.

10. Losno Garcia R, Santivañez V, Battilana CA. Singledose pharmacokinetics of ceftriaxone in patients with end-stage renal disease and hemodialysis. Chemotherapy. 1988;34(4):261-6. Erratum en: Chemotherapy 1989;35(3):following 235.

11. Elias A, Padron A, Ortiz M, Lavalle M, Battilana C, Rojas M, Koga V, Bustamante S. Study of irregular anti-N antibodies in the hematies of a renal transplant patient. Transplant Proc. 2002;34(1):363.

12. de Los Ríos R, Bussalleu A, Hurtado A, Valdivia J, Huerta Mercado J, Battilana C, Pala M, Pinto JL, Piscoya A, Cedrón H. [Prevalence of anti hepatitis $\mathrm{C}$ antibodies in patients with chronic renal failure receiving conservative therapy]. Rev Gastroenterol Peru. 2006;26(3):265-70.

13. Grados P, Battilana CA. Treatment of choleriform diarrhea during pregnancy. Bol Oficina Sanit Panam. 1994;116(3):198-203.

14. Grados P, Battilana C. Detection of convalescent Vibrio cholerae carriers using the enterotest. Bol Oficina Sanit Panam. 1994;116(4):285-9.

15. Grados P, Bravo N, Battilana C. Comparative effectiveness of co-trimoxazole and tetracycline in the treatment of Cholera. Bull Pan Am Health Organ. 1996;30(1):36-42.

16. Battilana CA, Lu L. Nefropatía diabética. Diagnóstico (Perú). 2000;39(2):66-79.

17. Battilana C. Valoración clínica del monitoreo ambulatorio de la presión arterial [editorial]. Diagnóstico [Internet]. 2001 [citado el 10 de diciembre de 2009];40(2). Disponible en: http:// www.fihu-diagnostico.org.pe/revista/index.html

18. Hinojosa R, Ige M, Battilana CA. Análisis del costo efectividad de dos inhibidores de la ECA en el manejo de la hipertensión arterial primaria: Cilazapril (inhibaceTM) vs. Captopril (genérico) en un modelo institucional. Diagnóstico (Perú). 2005;44(2):80-2.

19. Battilana CA. El linfocito B: Nuevo objetivo terapéutico en la artritis reumatoide. Diagnóstico (Perú). 2004;43(4):149-50.

20. Ige M, Hinojosa R, Battilana C. Ventajas de un buen manejo de la terapia adyuvante para cáncer de mama. Diagnóstico (Perú): 2007;46(1):34-6.

21. Hinojosa R, Battilana CA. Análisis fármacoeconómico del tratamiento adyuvante para el cáncer colorrectal en el Perú: Xelox vs. Folfox-4. Diagnóstico (Perú). 2005;44(3):116-8.

22. Hinojosa R, Battilana C. Costo global de los tratamientos para la artritis reumatoide. Diagnóstico (Perú). 2006;45(1):31-3.

23. Ige M, Hinojosa R, Battilana C. Manejo de las terapias para tratar a un paciente refractario con cáncer al púlmón de células no pequeñas: Análisis fármaco-económico y costo integral del tratamiento. Diagnóstico (Perú). 2006;45(2):80-2.

24. Salas A, Hinojosa R, Battilana CA. Costo de las terapias para tratar la insuficiencia renal crónica terminal (IRCT). Diagnóstico (Perú). 2006;45(4):182-4.

25. Ige M, Ruiz JE, Hinojosa R, Battilana C. Análisis fármaco económico entre Erlotinib y Pemetrexed en el tratamiento del cáncer de pulmón en segunda línea. Diagnóstico (Perú). 2007;46(2):68-70.

26. Ige M, Hinojosa R, Battilana C. Análisis farmacoeconómico de la terapia utilizada para el tratamiento de la hepatitis C. Diagnóstico (Perú). 2007;46(3):117-9.

27. Hinojosa R, Gómez M, Battilana CA. Análisis de costo efectividad del uso de Rituximab (MabtheraTM) como terapia de mantenimiento en el linfoma no Hodgkin indolente. Diagnóstico (Perú). 2008;47(3):129.31.

Manuscrito recibido el 10 de noviembre de 2009 y aceptado para publicación el 25 de noviembre de 2009.

\section{Correspondencia:}

Dr. José Carlos Pacheco Romero

Facultad de Medicina

Universidad Nacional Mayor de San Marcos

Av. Grau 755. Lima 1, Perú

Correo-e: jpachecoperu@yahoo.com 